\title{
EXTREME METEO-OCEANIC EVENTS
}

\author{
Franck Mazas ${ }^{1}$ and Luc Hamm ${ }^{1}$
}

\begin{abstract}
The two-step framework for over-threshold modelling of environmental extremes proposed in Bernardara et al. (2014) for univariate analyses is generalized to an event-based framework applicable to multivariate analyses. The distinction between sequential values (temporal observations at a given time step) and the event-describing values (such as storm peaks in univariate POT extrapolations) is further detailed and justified. The classification of multivariate analyses introduced in Mazas and Hamm (2017) is refined and linked to the meaning of the concepts of event, sampling and return period that is thoroughly examined; their entanglement being highlighted. In particular, sampling is shown to be equivalent to event definition, identification and description. Event and return period definition are also discussed with respect to the source phenomena or to response (or structure) phenomena. The extreme event approach is thus proposed as a comprehensive framework for univariate and multivariate analyses for assessing natural hazards, seemingly applicable to any field of environmental studies.
\end{abstract}

Keywords: event, sampling, return period, extreme values, Peaks-Over-Threshold, multivariate analyses

\section{INTRODUCTION}

Storm waves offshore, cyclones, coastal flooding, overtopping of harbor breakwaters, beach erosion, joint occurrence of storm surge with spring tide, resonance in ship mooring systems, etc., are examples of cases to be considered for design in port and coastal engineering. However this enumeration shows that these cases can imply one variable or several, concern environmental phenomena or loads on structures, be caused by extreme values or the combination of frequent ones. Despite this diversity, they are all meaningful as "something happening": they are events. Hence we wish proposing a clarification of the concept of meteo-oceanic events in order to provide the engineers with a sound framework for determining extreme conditions.

Meteo-oceanography, that is the gradual connection of two scientific disciplines, physical oceanography and marine meteorology, that have long evolved in parallel, is characterized by strong spatial and temporal variabilities, which makes it unsurprising that this field is active in setting up robust and sophisticated methodologies for estimating extreme values. In the univariate cases, the probabilistic methodologies have gradually converged towards the so-called "GPD-Poisson" model, that combines a Peaks-Over-Threshold (POT) declustering with a fit to a Generalized Pareto Distribution (GPD). The POT declustering was recommended as soon as the mid-1990's by the IAHR Working Group on Extreme Wave Analysis (Mathiesen et al., 1994), the storm peaks being fit to a Weibull distribution. Considering that the low of the exceedances over a threshold $u$ asymptotically tends to a GPD when $u$ is large enough (Pickands, 1975), the used of a GPD was quite logically associated to the POT declustering in the following years (Coles, 2001) and the GPD-Poisson model is now widely used for univariate extremes.

The authors already proposed improvements to this methodology. First, they advocated (Mazas and Hamm, 2011), a multi-distribution approach in order to complement the GPD with other distributions such as the Weibull, Gamma or Exponential laws, on the basis of daily practice of engineering and practical experience that the GPD often tends to underestimate the extreme values when the sample is not large enough (in other words, if the asymptotic domain is not reached yet). Second, they showed (Mazas et al., 2014a) that the conditions for the Maximum Likelihood Estimator to be valid are not met in a POT framework, and therefore proposed the use of the L-moments estimator (Hosking, 1990).

Last, they distinguished two separate steps for over-threshold modelling (Bernardara et al., 2014). The first step consists of event identification and characterization based on physical considerations, by setting up the i.i.d. sample to be analysed using the extreme value theory. Applying a POT declustering to the time series is the most common way to achieve this. The second step consists of using the statistical tools of the Extreme Value Theory (EVT) to determine the optimal threshold over which the peak exceedances are to be extrapolated by a statistical distribution.

The concept of physical event was thus introduced and justified in univariate analysis, and its link with the sampling of the time series, that is its declustering, was established. Still, this framework remained insufficient in the multivariate case, in particular for dealing with the interactions between variables such as wave height, wave period, sea level, current velocity or wind speed. An extension of the event framework to a bivariate and more generally multivariate dimension thus appeared to be necessary. This was begun $b$ considering the common case of the combination of sea level and wave 
height (Mazas and Hamm, 2017) and providing a methodology based on the modelling of dependence by extreme value copulas to derive the joint distribution of the variables and draw contours of the bivariate return period associated with the joint exceedance probability.

But beyond the choice of the copulas or the estimators to be used, a reflection was started on the sampling for multivariate analyses in meteo-oceanography. This reflection was deepened by considering other fields of natural hazards and through discussions with hydrologists struggling with the definition of extreme event for flash floods with torrential lava or debris flow occurring only a few times in a century, or linked to an extremely rare combination of primary causes. Questioning the terminology and the meaning of words and definitions was also a helpful approach. A comprehensive and sound framework on extreme events is thus proposed for assessing the extreme values of meteo-oceanic variables.

\section{A DETOUR ON TERMINOLOGY}

At first, physical concepts are to be reminded, as they will be used further. To begin with, a phenomenon, from the Greek phainein, "to show, shine, appear, to be manifest or manifest itself", can be defined as any thing which manifests itself and may be described as a system of information related to matter, energy or spacetime. Main examples of physical phenomena in meteo-oceanography include wind (a flow of gases), currents (a flow of liquid) or waves (fluctuations of free surface in a certain range of frequencies caused by the wind).

An important set of definitions is provided by the International Vocabulary of Metrology (VIM) from the Joint Committee for Guides in Metrology (JCGM, 2012). In particular, it specifies that a physical quantity is a "property of a phenomenon, body or substance, where the property has a magnitude that can be expressed as a number and a reference". So a physical quantity is a property that can be quantified by measurement, such as temperature or spectral significant wave height. Interestingly, the VIM also specifies that "in some definitions, the use of non-defined concepts (also called "primitives") is unavoidable. In this vocabulary, such non-defined concepts include: system, component, phenomenon, body, substance, property, reference..."

When a phenomenon is made of several components that happen on the same body, we thus deal with three concepts that are non-defined in this system of definitions. For instance, sea level fluctuations are the result of the superposition of many components associated with distinct physical phenomena: long-term variations in mean sea level, astronomical tide, meteorological surge, low-frequency waves, tsunamis, short waves. Each component corresponds to a particular phenomenon and can be described by physical quantities such as height and period (for periodic phenomena); their sum can be described by the physical quantity "sea level". According to the VIM, these components are all of the same kind. The kind of quantity refers to "the aspect common to mutually comparable quantities", though "the division of 'quantity' according to 'kind of quantity' is to some extent arbitrary".

In probabilities, we can also briefly recall a few basic definitions. A random variable is " $a$ variable quantity whose possible values are numerical outcomes of a random phenomenon" (Blitzstein and Hwang, 2014), random itself being a lack of predictability in these outcomes. In a random experiment, a single outcome is realized among a set $\Omega$ of possible outcomes, called the universe. A subset $A$ of $\Omega$ is called an event and its probability $\mathbb{P}_{A}$ is a measure of the likelihood that it will occur, defined as a weighting of this event relative to $\Omega$.

A trickier probabilistic concept is that of return period $\mathcal{T}$, first introduced in hydrology by Fuller (1914). Rather than the common but misleading definition of "the average period between two occurrences" of the event, it should rather be understood as a yearly probability of exceedance: every year, the probability that the $\mathcal{T}$-year value is reached or exceeded at least once is $1 / \mathcal{T}$. It can hence be linked to the probability of the event introduced above: $\mathcal{T}=1 / \lambda \mathbb{P}_{A}$, with $\lambda$ the mean number of occurrences per year. Thus defined as the probability of an event exceeding a value over a given duration, the return period seems to be unequivocal. However, it will later be seen that it becomes highly ambiguous in the multivariate case.

So far, definitions have been provided for the two fields of physics and probabilities. In order to join them, the key concept is that of event: what exactly is this subset of all the possible outcomes of a random experiment, and how can it be interpreted? Is there a choice to make that will affect the meaning of the return period?

The English word "event", from the Latin evenire "to come out, happen, result", has had two distinct meaning over the time: first as "the consequence of anything", then as "that which happens". In the famous Encyclopaedia of Diderot and d'Alembert, Louis-Jacques Goussier wrote in 1756 a definition where these distinct meanings of the outcome of "something" or this "something" itself appear, with this 
following interesting development for the latter one: "any remarkable circumstance that is determined over time of all contingent things. But may this word be a radical of the language itself, so necessary for defining the other words that it is hardly definable itself?" This last sentence obviously echoes the "primitives" mentioned by the VIM.

Last, let us notice that the ISO/Guide 73:2009 provides a vocabulary relative to risk management with definitions of events that are interesting in terms of their approach to risk. It specifies that "risk is often characterized with reference to potential events and consequences, or a combination of these" and that "risk is often expressed in terms of a combination of the consequences of an event (including changes in circumstances) and the associated likelihood of occurrence". An event is then defined as an "occurrence or change of a particular set of circumstances".

In modern times, the meaning of "something occurring" is definitely accepted and the oldest sense of the outcome or consequence no longer holds, but all fields, from metrology to etymology, insist on the need to precise the event and to delineate its definition: a conclusion whose application is the core of this paper.

\section{UNIVARIATE EVENTS}

In the univariate case, the two-step framework introduced by Bernardara et al. (2014) provides a relevant event-based framework for over-threshold modelling of environmental time series (Figure 1). It stems from the observation that the analyst generally deals with a time series of observations of the variable at a given time step, while the conventional tools provided by the EVT assume that the dataset is independent and identically distributed (i.i.d.). Consequently, a first step of declustering is needed to extract the i.i.d. peaks from the time series. Though considering the exceedances over a threshold can be viewed as a practical tool to achieve this extraction, the significance of this operation goes deeper.

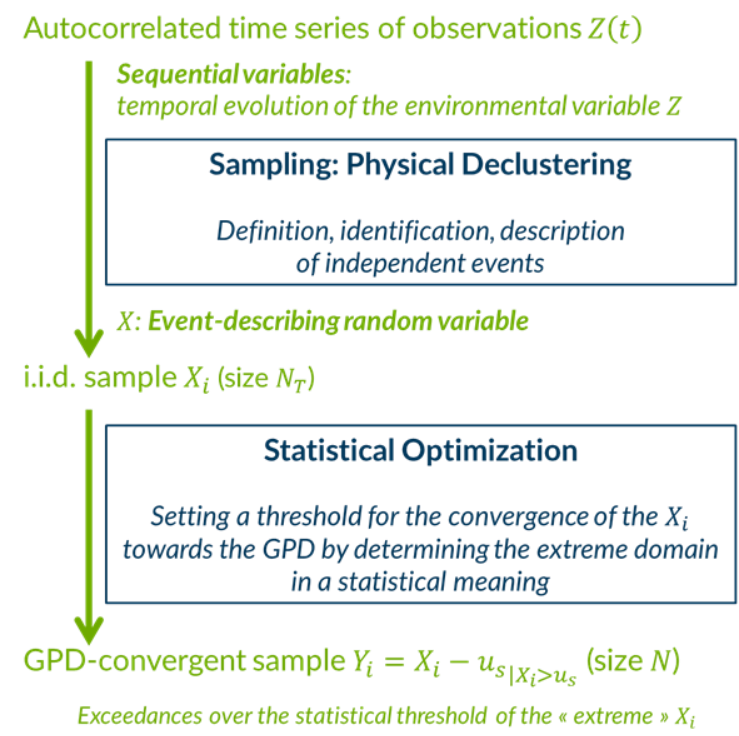

Figure 1: Two-step framework for over-threshold modelling, after Bernardara et al. (2014)

The time series provides discrete values, at a given time step, of a continuous environmental variable, i.e. discrete observations of a physical quantity describing a physical phenomenon, such as wave height or wind speed. The basic laws of physics are such that these quantities are temporally autocorrelated. The finer the time step of the series, the stronger the correlation. So setting a threshold will not extract individual values: it will identify time intervals within which the observation is far from its average value: in other words a storm, a flood, etc. These anomalies have a duration and a magnitude, and the peak is a very partial description of these.

This it appears that one can no longer reason only in terms of discrete values. It has been identified "something that happens" "over time" to a physical quantity, linked to physical phenomena: in other words, as seen above, a physical event. And so far it has been chosen, very simply, to describe this event in terms of the maximum value or the associated quantity: the peak value.

This yields another consequence: the peak value is a different kind of variable than the variable observed at each time step. This is no longer the quantification of a physical quantity but a description of a physical event. Besides, other descriptions could be imagined, such as the average value over the duration of the event, or temporal integration. This leads to introducing some new terminology: 
- the time series is made up of sequential variables, i.e. discrete observations of the temporal variations in the environmental variable;

- $\quad$ the i.i.d. sample is made up of event-describing variables (most of the time, event peaks).

To sum up, this first-step processing is a physical declustering with the aim of identifying and describing independent events, by extracting an i.i.d. sample of the event-describing variable from the autocorrelated time series of sequential values. The threshold associated to this step is thus named "physical threshold $u_{p}$ ".

Note that a threshold is not always necessary for event identification. For instance, cyclonic studies can be carried out by modelling the sea states generated by the cyclones, each of which is archived by the cyclonic warning centres. There is no continuous time series but a set of already clearly identified events. Selecting the maximum wave height generated by the cyclonic atmospheric fields is enough to set up the i.i.d. sample.

Once this i.i.d. sample has been set up, a new step of statistical optimization can begin to find the best statistical model to fit to the data thanks to the Extreme Value Theory, and the reasoning becomes purely statistical. In the framework of extreme estimation through over-threshold modelling, the threshold can thus be called "statistical threshold $u_{s}$ ". Physical arguments are of no use here, with the possible exception of recommendations about the mean number of events per year.

At this stage, the complementary role of physical and statistical approaches has been shown, the distinction between the sequential observations and the event-describing variable has been highlighted and univariate events may be defined as an anomaly of a physical quantity that takes values far from the average over a certain period, described by a distinct quantity.

Besides, although the physical quantity of the sequential observations describes a physical phenomenon (e.g. wave height for sea states, wind speed for wind, etc.), it is also quite legitimate to consider the event itself as a physical phenomenon (a storm, flood, etc.). Therefore, even in the simple univariate case, the non-definability of the concepts of event, phenomenon etc. referred to hereinabove can be experienced.

\section{A NEW CLASSIFICATION FOR MULATIVARIATE ANALYSES}

In meteo-oceanography, it is quite common to consider more than the mere peak of a single variable. The role of storm duration for cumulative damage on a breakwater; the separate analysis of astronomical tide and meteorological surge for assessing extreme sea levels; the combined effect of waves and sea level for beach erosion or wave overtopping of coastal structures: these are just some of the issues potentially called into play, and for which a methodology is required in order to estimate the extreme joint probabilities of several physical quantities.

Still it can be seen that these cases will not be handled in the same way. Storm duration describes the event only and has no sequential equivalency, as opposed to the peak wave height (event-describing variable) that is the maximum of the time series of, say, hourly wave height (sequential variable) during the storm. A bivariate analysis of astronomical tide and meteorological surge can be made without appealing to joint distributions: they be analysed separately then recombined by a convolution operation (Mazas et al., 2014b) into a single variable that is sea level. In contrast, the sum of significant wave height and wind speed (two quantities of different dimension) but also that of wind speed and current velocity (two quantities of the same dimension) are meaningless: these physical quantities are not of the same kind.

More generally, clear distinctions in bivariate analyses that are directly linked to what is called events can now be seen. Making use of the definitions recalled above, the following classification can be proposed for multivariate events:

- $\quad$ Type A: a single phenomenon described by different physical quantities that are possibly not of the same kind;

- $\quad$ Type B: a single phenomenon comprised of different components, described by physical quantities of the same kind between one component and another;

- $\quad$ Type C: several phenomena described by physical quantities that are possibly not of the same kind. 
- Type A: a single phenomenon described by different physical quantities that are possibly not of the same kind

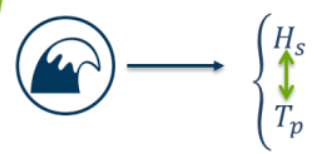

- Type B: a single phenomenon comprised of different components, described by physical quantities of the same kind between one component and another

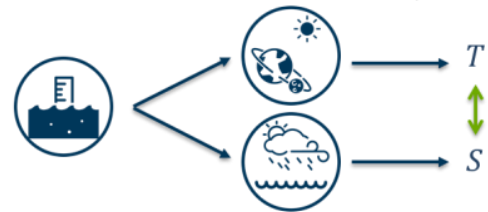

- Type C: several phenomena described by physical quantities that are possibly not of the same kind

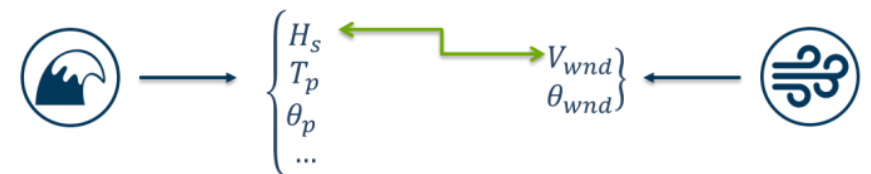

Figure 2: Illustration of the proposed classification for multivariate analyses

The point of this classification consists in its implications when it comes to sampling. Furthermore, sampling is also intimately linked with dependence, which lies at the core of multivariate analyses, because dependence is assessed on the sample. Sampling itself is to be considered as event definition, identification and description, as will be shown hereafter.

\section{SAMPLING: A DESCRIPTION OF EVENTS}

In Type A analyses, sampling is generally straightforward. When dealing with directional extreme winds or $H_{s} / T_{p}$ correlation, it is intuitive to define the event as an anomalous value of the quantity that measures the energy most directly, such as wave height, wind speed or current velocity. The choice of the event-defining variable is easily made and the other variable is a mere covariate; the value concomitant to the peak of the event-defining variable is generally taken, unless this other variable describes the event as a phenomenon itself. This is in particular the case of event duration that may play a key role in the analysis: storm duration is cause of damage progression on rubblemound breakwaters (Melby and Kobayashi, 1998). Figure 3 illustrates such a sampling where the event is defined as large values of $H_{s}$, identified through exceedances of $H_{s}$ over a threshold, and described by the peak $H_{s}$, the concurrent $T_{p}$ value and the storm duration.

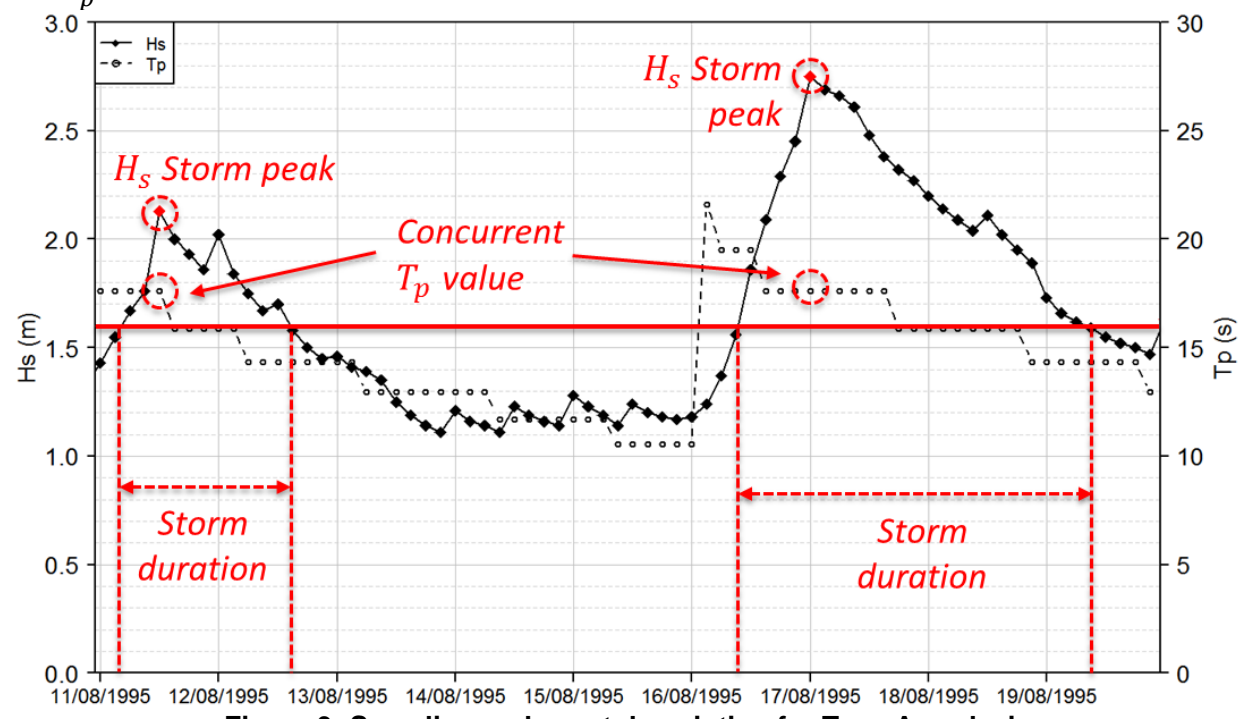

Figure 3: Sampling and event description for Type $A$ analysis

In Type B analyses, the components can be dealt with separately before being reduced to a single variable (the general phenomenon), and sampling can be performed on each component within the univariate framework described in Figure 1 (see details in Mazas et al., 2014b). 
Things become much trickier in Type-C analyses. For instance, both wave height and wind speed are closely linked to energy and there is no obvious choice for setting an event-defining variable. In addition, it must be borne in mind that the joint occurrence of two (or more) phenomena is usually required because their combination causes a distinct phenomenon: beach erosion, wave overtopping, coastal flooding, etc. Furthermore, these response phenomena do not necessarily require the joint occurrence of large values of the source phenomena: the combination of a large value of one with a frequent value of the other may generate an event as far as the response phenomenon is concerned.

It thus begins to appear that a point of view needs to be chosen. This also explains the variety of sampling methods found in the literature. Generally speaking, they differ in the answer they offer to the following question: should the method focus on the exceedance of one variable only, of both, or of one or the other? We give below three examples of sampling methods, among many others.

Wahl et al. (2016) considered their events as waves and sea level conditions likely to cause dune erosion. Thus they defined and identified them as wave height exceedances over a threshold, then described them by the peak wave height, the concurrent wave covariates (period $T_{p}$, direction $\theta$ ) and the maximum value of non-tidal residual $\eta_{N T R}$ during the storm (Figure 4): the event-defining variable is there $H_{s}$. High tide sampling for wave / sea level analyses in a macrotidal environment, which consists in selecting the sea level at high water along with the concomitant $H_{s}$ value, is based on the postulate that the analysis is performed for phenomena that can only occur at high tide: in this case, the eventdefining variable is sea level. Such a choice of event definition would not be appropriate when assessing the hazard of loss-of-coolant situation in a nuclear power plant cooled by seawater, a case for which low tide sampling could be chosen. A bivariate threshold tries to consider both variables equivalently by selecting pairs that correspond to large values for both phenomena: there are two event-defining variables.

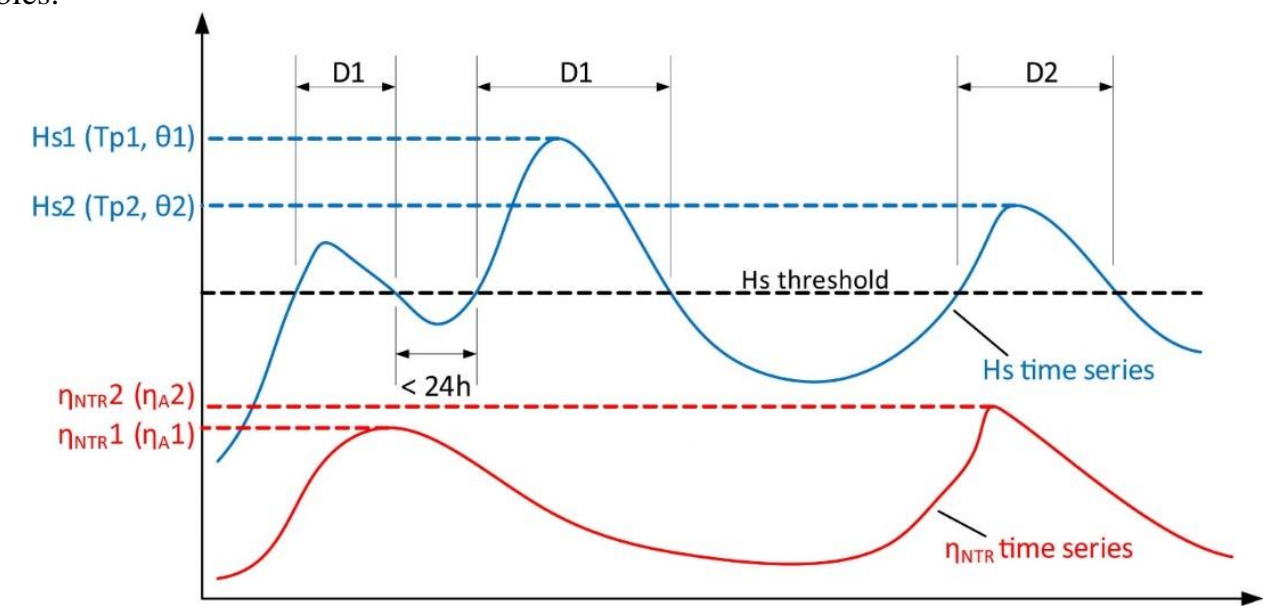

Figure 4: Storm definition for a waves / sea level analysis, after Wahl et al. (2016)

A fourth example of multivariate sampling is given in Mazas and Hamm (2017) in order to account for covariates such as wave period or direction: the response function method. The idea is to combine the variables and their relevant covariates in a univariate function, on which a univariate POT declustering can be applied. The multivariate event is there identified by the peaks of this response function, and described by the values of the variables concurrent to these peaks (Figure 5).
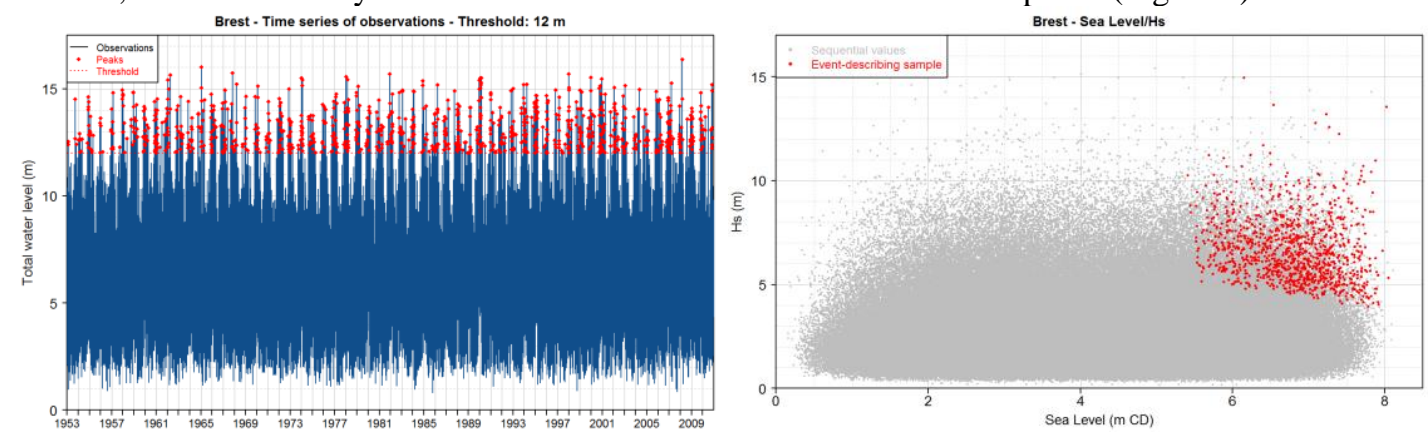

Figure 5: Left: time series of a univariate response function (sum of sea level and nearshore wave height) with threshold and peak of the selected events; right: sequential pairs (Hs, Z) of the time series (in grey) and selected event-describing pairs (in red) 
An important change is that the event-describing variable of the univariate framework, most of the time a peak, is now an event-describing tuple, or pair in the bivariate case. Once again, it may be that none of its elements is a peak of its variable.

The three dimensions of sampling, namely event definition, identification and description, can thus be illustrated with one of the aforementioned methods: high tide sampling. It is clear that choosing this sampling necessarily defines an event as what happens at high tide, and vice-versa. Identification consists in finding the maxima of the variable "sea level". Last, the description of such events requires choices: instantaneous surge at the time of the high tide or skew surge, wave height at the time of the high tide or its maximum value within a time window centered on the high tide, and so on.

Once the event-describing sample is set up, the events are modelled as follows: assessment and modelling of the dependence between the variables $X$ and $Y$, modelling of the marginal distributions $F_{X}$ and $F_{Y}$, and last derivation of the joint probability distribution $H_{X, Y}$ (see details in Mazas and Hamm, 2017).

\section{RETURN PERIOD IN MULTIVARIATE ANALYSES}

Once the multivariate sample of the i.i.d. event-describing tuples is set up, the joint distribution of the variables can be assessed using more or less sophisticated approaches. However, the correct interpretation of the output of the analysis is still at stake.

As seen hereinabove, the return period is the yearly probability of the event, defined as the exceedance of a value in the univariate case. Still, two variables offer a wider range of possible combinations to define the event $A$, i.e. the subset of all possible outcomes, and its probability $\mathbb{P}_{A}$. These combinations include: the joint exceedance of the two variables $p_{A N D}=\mathbb{P}[X>x \cap Y>y]$, the exceedance of at least one of the variables $p_{O R}=\mathbb{P}[X>x \cup Y>y]$, various conditional probabilities, and so on. All these probabilities are different and therefore the associated return periods will be different. But even though it can be written, for instance, $p_{O R} \geq p_{A N D}$, this comparison is somewhat meaningless as it is a comparison of different events.

There again a choice thus has to be made between these different probabilities, depending on the aim of the study. As seen above, choosing a probability means choosing an event, and choosing an event means choosing a sampling: event, sampling and return period are totally entangled concepts.

This choice actually has to be made between the source variables and the possible response variables: the conditions of the probability (the definition of event $A$ ) may no longer depend on the source variables $X$ and $Y$, but on a response variable $Z=g(X, Y)$, where $g$ is a function that links the source (environmental forcing) variables $X$ and $Y$ to one specific response (structural) variable $Z$. It can be seen that this is a reduction from the bivariate case back to the univariate definition of the probability of exceedance and the associated $\mathcal{T}$.

When it comes to the joint return period of the source variables, it is generally the return period associated to the probability of joint exceedance $\mathcal{T}_{A N D}=1 / \lambda p_{A N D}$ that is chosen, as illustrated in Figure 6.

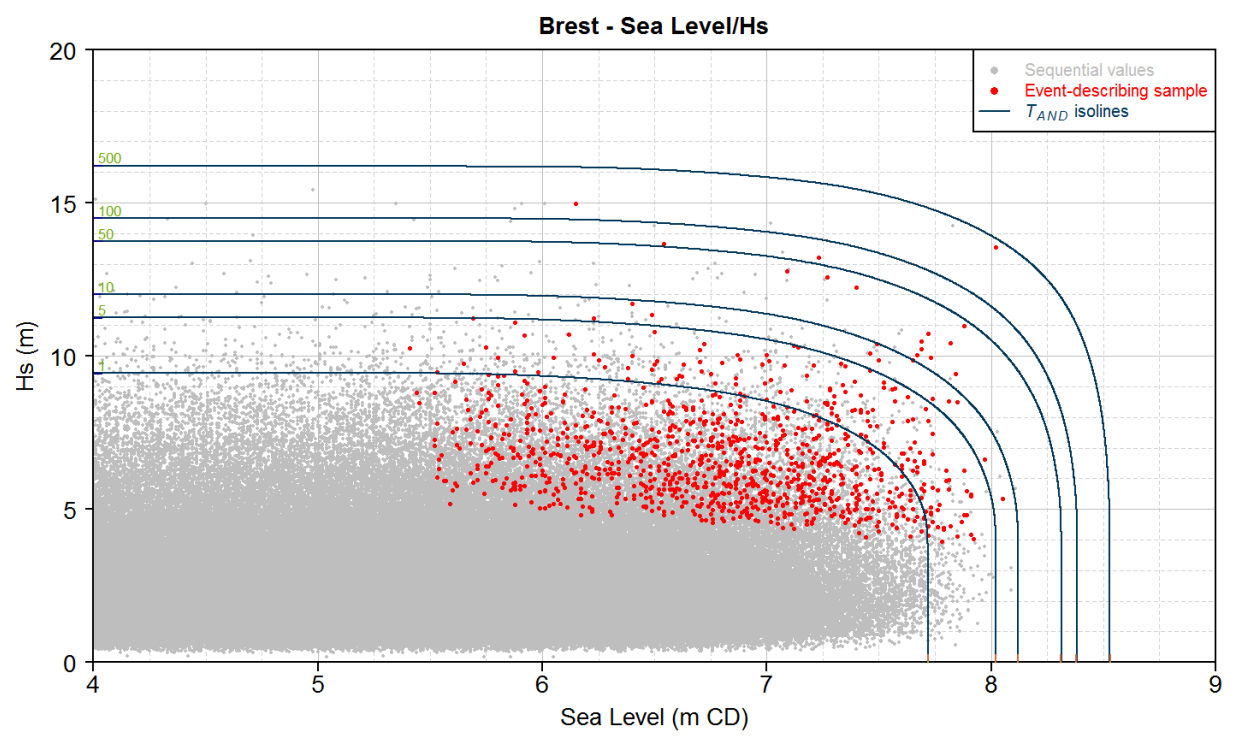

Figure 6: Contours of return period of joint exceedance for wave height and sea level 
The return period $\mathcal{T}_{S}$ associated with the response function (sometimes called structure variable) is different from the joint return period associated with the source variables $\mathcal{T}_{\text {AND }}$. It is generally lower, as illustrated by the following simple example based on $H_{s}$ and sea level, but without having to account for wave covariates (more "useful" structure variables such as wave overtopping also depend on wave period, direction, etc): the response function $H_{s}+Z$. The extreme values can be extrapolated directly for this return function and drawn as contour lines of $\mathcal{T}_{s}$ in the $\left(H_{s}, Z\right)$ plane (Figure 7$)$. A colour code based on these extreme values is also applied to the event points. The contours of the return period of the response functions, that are straight lines in this simple example, are almost tangential to the contours of equal joint return period $\mathcal{T}_{A N D}$. When running along a contour of the bivariate return period of the source variables, the univariate return period of the response variable is lower, and equal (or close) on a single point, that is a worst case for this particular response function. Should another response function be chosen, this worst case point would be located elsewhere on the contour.

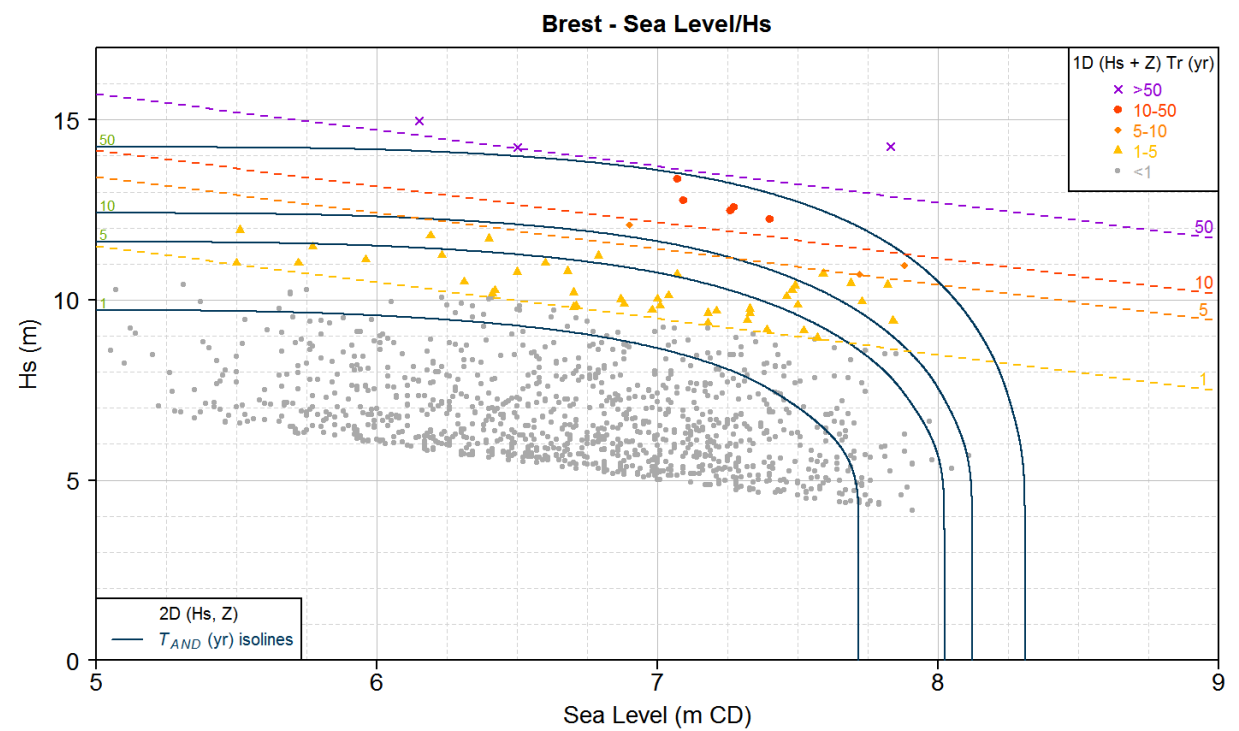

Figure 7: Contours (plain blue) of joint exceedance probabilities (Hs, Z) and isolines (dashed) of univariate return period of the response function $(\mathrm{Hs}+\mathrm{Z}$ )

\section{CONTOURS OF RETURN PERIOD FOR EVENT-DESCRIBING VALUES}

It may be requested in some engineering studies to draw bivariate contours for return periods of 1 , 10,100 years or more. This is generally on account of a need to define a large number of load cases for the design of the structures. Because there may be different kinds of loads to study, it is generally implicit that the return period refers to the source variables, and that it consists of $\mathcal{T}_{A N D}$.

Return period was defined hereinabove as the inverse probability of exceedance (univariate case) or as a joint or conditional exceedance (multivariate case), expressed in years using the Poisson parameter. A bivariate contour is related not to a notion of probability of exceedance, but to one of probability of occurrence, which is quite different. Then it should be made clear, in spite of the misuses of language that can be found in the engineering studies or in the technical specifications of tenders, that contours of return period cannot be drawn per se: the link is rather indirect.

A proposed answer consists in drawing the contour associated with the quantile of the given return period of one of the source variables, named the reference variable. This kind of contour has been proposed by other authors (Galiatsatou and Prinos, 2007) but the need to choose the reference variable does not appear clearly. More specifically, for a return period of $\mathcal{T}$ years and with $Y$ being the reference variable, the method is as follows. First, the $\mathcal{T}$-year quantile $y_{\mathcal{T}}$ of the reference variable is determined from its marginal distribution $F_{Y}$. Then the most probable value of $X$ given $Y=y_{\mathcal{T}}$ is selected: this is the maximum of the joint density of $X$ and $Y$ along the line $Y=y_{\mathcal{T}}$. A contour of the density $p_{X, Y}\left(x_{\max }, y_{\mathcal{T}}\right)$ can then be drawn in the $(X, Y)$ plane.

This approach is illustrated in Figure 8 for a bivariate analysis $H_{s} / Z$. Dotted dark blue lines are the contours of joint density of the event-describing pairs $H_{S} / Z$ calculated from the most probable values of $Z$ for the marginal extreme quantiles of $H_{s}$, represented by the horizontal dot-dashed blue lines.

But because this is a Type-C analysis, the reference variable could be chosen to be the sea level $Z$ : this would yield the plain brown contours of iso-density from the marginal extreme quantiles of $Z$, 
represented by the vertical dashed brown lines. In a Type-A analysis, the reference variable is generally clearly identified.

These two sets of contours are drawn from the exact same joint distribution $H_{X, Y}$, just as a univariate distribution can be illustrated by its density, its cumulative distribution function or its survival function. It can also be understood from this figure that the flat (horizontal or vertical) parts of the contours of $\mathcal{T}_{A N D}$ (see Figure 6) correspond to areas of nil joint density, where the probability of joint exceedance $p_{A N D}$ does not vary along the contour.

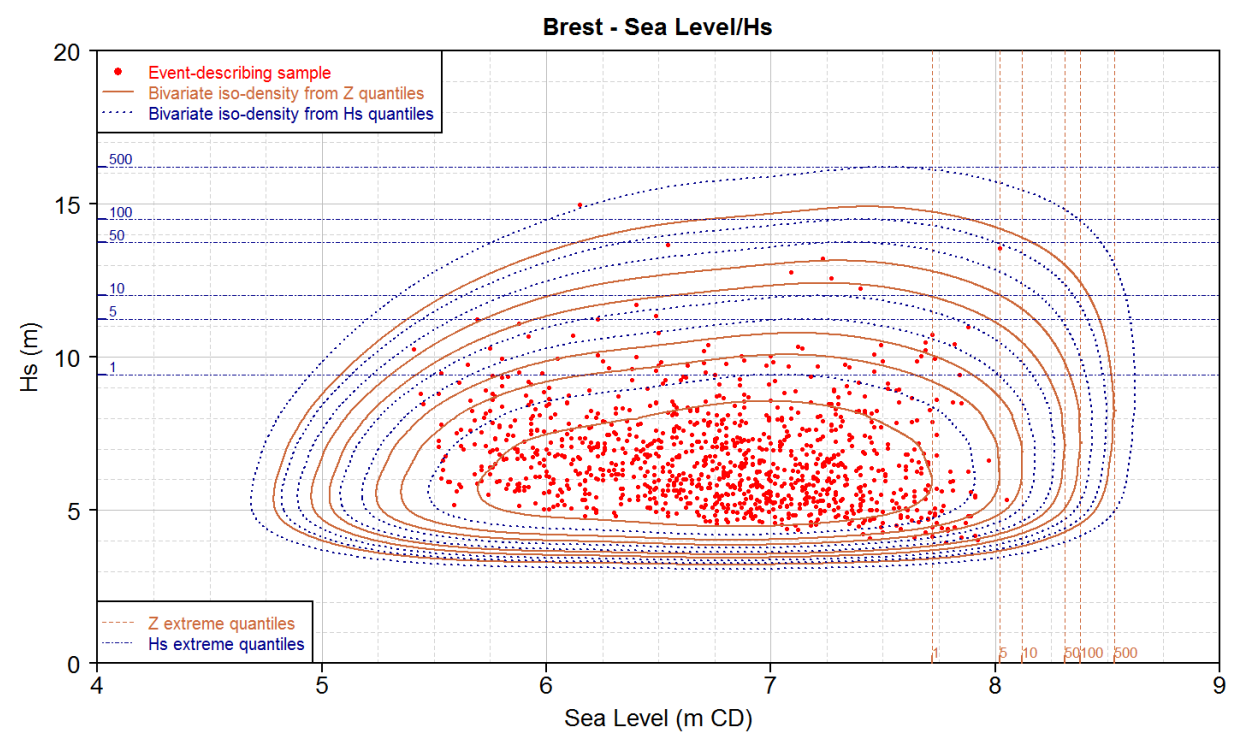

Figure 8: Contours of Hs / sea level joint return periods

\section{FROM EVENT-DESCRIBING SAMPLE TO SEQUENTIAL VALUES}

Drawing contours of joint density of the event-describing sample linked to the univariate return period of the marginal distributions makes quite naturally arise the idea of drawing such contours over the full range of values that the variables can take, rare or frequent. In other words, is it possible to use the joint distribution of the events to derive the joint distribution of the sequential values, such as hourly observations of sea states and sea level? This request is often motivated by the need to define a large number of load cases for the design of offshore or coastal structures such as offshore wind turbines.

A parallel can be made with the univariate mixture model proposed in Mazas et al. (2014) to derive the distribution of the sequential surges. First, parametric distributions are used to model the peak exceedances (possibly for both tails). These conditional distributions of surge peaks are then transformed into conditional distributions of sequential surges beyond the threshold that delineates the parametric domain. This transformation from the event-describing peak to the sequential observation is made possible by the use of an extremal index (Leadbetter, 1983) that may be interpreted in a first approach as the reciprocal of the mean number of sequential values per event (in other words, of the mean duration of the event). Last, the parametric tails are connected to the bulk of the distribution (frequent values of surges) modelled by an empirical distribution from a non-parametric kernel density estimator.

This univariate mixture model is adapted to the bivariate case, though somehow roughly. A bivariate parametric tail domain is defined on the upper-right corner of the $(X, Y)$ plane. Within this domain, the joint density of the event-describing pairs is transformed into the conditional joint density of the sequential pairs belonging to this domain by a simple extremal index, assumed constant and equal to the reciprocal of the mean number of sequential values per event. In other words, each event is assumed to have the same duration and therefore the conditional distribution of the sequential pairs in the parametric tail domain is identical to the distribution of the event-describing pairs. Then this conditional distribution (the sum of its density is 1) is multiplied by a factor equal to the ratio of the number of sequential pairs belonging to this domain over the total number of observations. Last, this parametric tail distribution is connected to the empirical bulk distribution of the frequent pairs, fitted by a kernel density estimator.

This preliminary approach is illustrated by the joint occurrence of wave height $H_{m 0}$ with wind velocity $W_{s}$, a Type-C analysis for which any of these variables can be seen as the event-defining variable. An event may be considered as large wave heights with the concurrent wind velocity and the eventdescribing sample set up by performing a POT declustering of the time series of $H_{m 0}$. In order to draw 
the contours of iso-density, the event-defining variable is naturally chosen as the reference variable (Figure 9, left). Conversely, an event may consist of large wind speeds with the associated sea states (Figure 9, right). Therefore, the event definition being different, the joint distribution is not identical between the two plots: the choice of the event definition and of the sampling will yield different contours related to the return period, even for the joint distribution of the sequential pairs: the entanglement between the concepts of event, sampling and return period is highlighted there again.
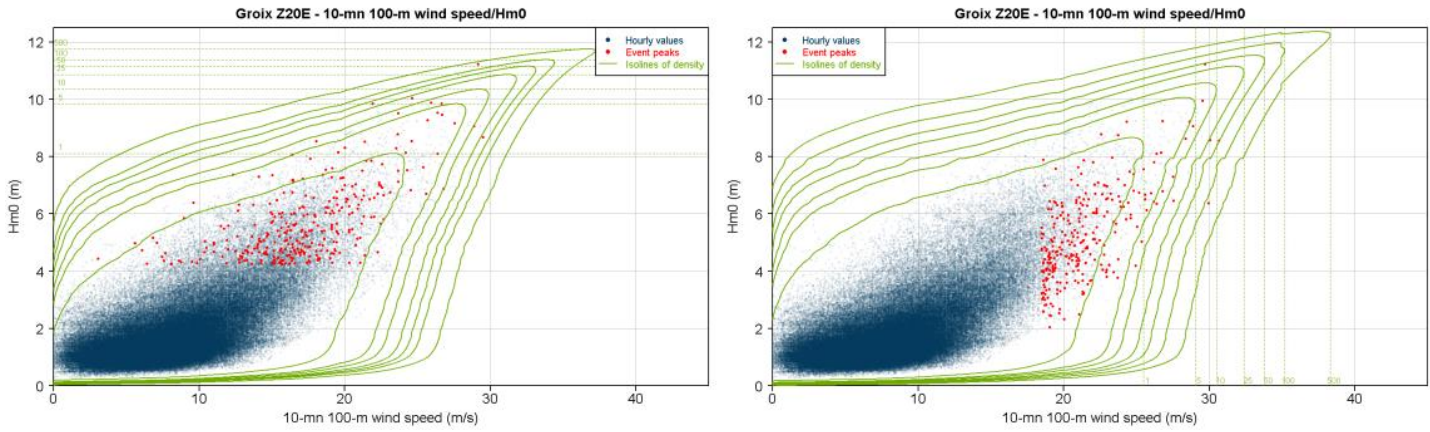

Figure 9: Contours of sequential $\mathrm{Hmo} / \mathrm{Ws}$ density associated with extreme $\mathrm{Hm} 0$ peaks (left) and extreme wind speed peaks (right)

\section{DISCUSSION AND CONCLUSIONS}

The present paper aims at highlighting the subjectivity of any analysis of extreme events, which is deemed intrinsic to such analyses. It wishes to stress how the choices made in the first steps of a study drive the meaning and interpretation of the results, far more than more technical issues such as the choice of a distribution or of an estimator. In brief, it attempts to clarify the relationships between key concepts of extreme studies and the implications of these relationships for a better use and application in hazard assessment and protection design.

This intrinsic subjectivity has been pointed out in different ways. The basic definitions in the fields of metrology, probability, risk assessment and even etymology all converged on the "radical", or "primitive" nature of the event and its requirement to be defined.

In particular, it should always be made clear in engineering whether the design criteria are to be based upon the source variables or the response function. This will drive the choices of event, sampling and, possibly, methodology. Indeed, the quasi-mechanical link between event definition and sampling method is the main topic this paper investigates. A physical point of view is advocated on that matter, which yields the proposed classification based on the distinction on physical phenomena that aims at guiding the analyst on his choice for the sampling method.

The pitfalls related to the return period are also addressed and there again this paper tried to bring clarifications and highlight how closely related to event definition and sampling is return period.

The distinction between the event-describing variable and the sequential values, already important in the univariate case, appears to be even more relevant in the multivariate case. This is in particular the case when closed contours (sometimes called environmental contours) are requested. However, the method proposed for deriving the bivariate mixture distribution of the sequential values from the parametric bivariate distribution of the event-describing sample is still exploratory and requires refinement in further work.

Considering all the above, it appears that only generic guidelines and recommendations can be addressed to extreme analysts. First and foremost is the necessary awareness that any study of extreme events is not a neutral tool of data analysis but a perceptual construct to be built from a physical understanding to reach a particular goal. It is thus recommended first to know and keep in mind the final aim of the study; and to understand the physical phenomena that are at work, how they interact with each other, how they are described. On that basis, using possibly the proposed classification and examples presented above, the appropriate choice of event definition and sampling method can be chosen, and the probability associated to the return period properly understood. Last, it will be decided whether it is necessary to incorporate the extrapolation of the extreme events into the distribution of the sequential observations.

In conclusion, this works insist on the intimate link between sampling, event, and the definition adopted for the return period, probability of exceedance or probability of occurrence. In particular, sampling should always be considered as "event definition, identification and description". 
These steps cannot be automated in a universal method but must be tailored completely to the final objectives of the study in hand, which is precisely what is expected of an engineer. This is why the event definition can be stated to be a matter of point of view.

The event approach is thus proposed as a comprehensive framework for univariate and multivariate analyses for assessing natural hazards, seemingly applicable to any field of environmental studies. It could be further developed in various ways, such as spatial extension, incorporation of historical information, or estimation of uncertainties. Still, the concepts and classifications presented here lay the groundwork for determining fully multivariate analyses that would include, in a single joint distribution, the different physical quantities describing distinct phenomena: a fine challenge for mathematicians who are aware of the beautiful complexities of the physics of extreme natural events.

\section{REFERENCES}

Bernardara P., F. Mazas, X. Kergadallan, and L. Hamm. 2014. A two-step framework for over-threshold modelling of environmental extremes, Nat Hazards Earth Syst Sci 14, 635-647.

Blitzstein, J., Hwang, J., 2014. Introduction to Probability. CRC Press.

Coles, S.G., 2001. An Introduction to Statistical Modeling of Extreme Values. Springer, London.

Fuller, W.E., 1914. Flood flows. Transactions of the American Society of Civil Engineers 77.

Galiatsatou, P., Prinos, P., 2007. Bivariate models for extreme of significant wave height and period. An application to the Dutch Coast. Proceedings of the 2nd IMA Conference on Flood Risk Assessment, 2007, Plymouth, Institute of Mathematics \& its Applications, pp.77-84.

JCGM, 2012. Joint Committee for Guides in Metrology - The International Vocabulary of Metrology Basic and General Concepts and Associated Terms, 3rd ed., JCGM 200:2012.

Leadbetter, M. R., 1983. Extremes and local dependence in stationary sequences. Probab Theory Rel, 65, 291-306.

Mathiesen, M., Goda, Y., Hawkes, P.J., Mansard, E., Martin, M.J., Peltier, E., Thompson, E.F., Van Vledder, G., 1994. Recommended practice for extreme wave analysis. Journal of Hydraulic Research 32, 803-814.

Mazas F. and L. Hamm. 2011. A multi-distribution approach to POT methods for determining extreme wave heights, Coastal Engineering 58, 385-394.

Mazas F., X. Kergadallan, P. Garat, and L. Hamm. 2014a. Applying POT methods to the Revised Joint Probability Method for determining extreme sea levels, Coastal Engineering 91, 140-150.

Mazas, F., Kergadallan, X., Garat, P., Hamm, L., 2014b. Applying POT methods to the Revised Joint Probability Method for determining extreme sea levels. Coastal Engineering 91, 140-150.

Mazas, F., Hamm, L., 2017. An event-based approach for extreme joint probabilities of waves and sea levels. Coastal Engineering 122, 44-59.

Melby, J. A., Kobayashi, N., 1998. Progression and variability of damage on rubble mound breakwaters. Journal of waterway, port, coastal, and ocean engineering, 124(6), 286-294.

Pickands, J., 1975. Statistical inference using extreme order statistics. Annals of Statistics 3, 119-131.

Wahl, T., Plant, N. G., Long, J. W., 2016. Probabilistic assessment of erosion and flooding risk in the northern Gulf of Mexico. Journal of Geophysical Research: Oceans, 121(5), 3029-3043. 